\title{
Risk and Incidence of Infection with Bevacizumab in Non-Small-Cell Lung Cancer Patients: A Meta-Analysis
}

\author{
Ling Peng $^{a}$ Bao-Dong Qin ${ }^{b}$ Song Xu ${ }^{c}$ Yang Xia $^{d}$ Jin-Song Yang ${ }^{e}$ Kui Xiao $^{f}$ \\ Justin Stebbing ${ }^{9}$ \\ ${ }^{a}$ Cancer Center, Department of Pulmonary and Critical Care Medicine, Zhejiang Provincial People's Hospital,

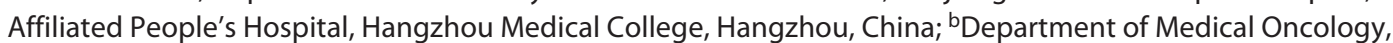 \\ Changzheng Hospital, Shanghai, China; 'Department of Lung Cancer Surgery, Lung Cancer Institute, Tianjin \\ Medical University General Hospital, Tianjin, China; ${ }^{\mathrm{d} D e p a r t m e n t}$ of Respiratory and Critical Care Medicine, Second \\ Affiliated Hospital of Zhejiang University School of Medicine, Hangzhou, China; ${ }^{\text {eDepartment }}$ of Radiotherapy, The \\ First Affiliated Hospital, School of Medicine, Zhejiang University, Hangzhou, China; 'Department of Pulmonary and \\ Critical Care Medicine, The Second Xiangya Hospital, Central South University, Changsha, China; ${ }^{9}$ Division of Cancer, \\ Department of Surgery and Cancer, Imperial College London, London, UK
}

\section{Keywords}

Bevacizumab · Infection · Meta-analysis · Non-small-cell lung cancer $\cdot$ Risk

\begin{abstract}
Background: A previous meta-analysis suggested that use of bevacizumab is associated with an increased risk of infection in cancer patients. With the continuous accumulation of evidence in non-small-cell lung cancer (NSCLC), we performed a new, focused meta-analysis of randomized controlled trials (RCTs) to quantify the relative risk (RR) and incidence of infectious complications in those individuals treated with bevacizumab. Methods: Electronic databases were searched, including PubMed, Embase, and Cochrane databases. Eligible studies were prospective randomized clinical trials of NSCLC patients treated with bevacizumab with toxicity data on infectious complications. RR, overall incidence rates, and 95\% confidence intervals $(\mathrm{Cl})$ were calculated using fixed- or random-effects models, depending on the heterogeneity of the included studies. Results: A total of 4,545 patients from 14 prospective RCTs were included in the meta-analysis. Treatment with bevacizumab was not associated with an increased risk of all-grade (RR 1.12, 95\% Cl: 0.84-1.49) or high-grade (RR 1.11, 95\% Cl: 0.86-1.41) infections, respectively. The summary incidences of all-grade and high-grade infections in patients receiving beva-
\end{abstract}

Karger@karger.com www.karger.com/ort

Karger ${ }^{\prime \prime}=$ BOPEN ACCESS
(C) 2022 The Author(s)

Published by S. Karger AG, Basel

This is an Open Access article licensed under the Creative Commons Attribution-NonCommercial-4.0 International License (CC BY-NC) (http://www.karger.com/Services/OpenAccessLicense), applicable to the online version of the article only. Usage and distribution for commercial purposes requires written permission. cizumab in the treatment group were $16.4 \%(95 \% \mathrm{Cl}$ : $15.7-$ $17.2 \%$ ) and $4.3 \%$ (95\% Cl: 3.0-6.1\%), respectively. Conclusions: The use of bevacizumab is not associated with a significantly higher risk of infections in NSCLC patients. These data provide reassurance regarding the risk of infection in patients with NSCLC receiving bevacizumab.

(c) 2022 The Author(s).

Published by S. Karger AG, Basel

\section{Introduction}

Among the proangiogenic factors, the most studied factor for therapeutic application is the vascular endothelial growth factor (VEGF). Antiangiogenic drugs have been shown to result in regressions of normal capillaries and blocking new blood vessel formation [1]. Bevacizum$\mathrm{ab}$, a humanized monoclonal antibody that binds to the VEGF receptors, has been approved for the treatment of many advanced cancers, including colorectal cancer, non-small-cell lung cancer (NSCLC), breast cancer, renal-cell carcinoma, and glioblastoma multiforme. It is generally well tolerated for the majority of patients. Common adverse effects include hypertension, proteinuria, and gastrointestinal symptoms. However, excessive toxicity may necessitate dose reductions or treatment cessation, which may limit therapeutic efficacy. 
Little attention has been paid to infectious complications associated with the use of bevacizumab. Clinical trials have reported a substantial variation in the risk and incidence of infection and febrile neutropenia related to bevacizumab [2]. A previous meta-analysis published 5 years ago including 33,526 patients from 41 randomized controlled trials (RCTs) suggested that bevacizumab is associated with an increased risk of infection in cancer patients [3]. However, there were only 4 RCTs of NSCLC included in the meta-analysis, which may not confidently inform about the risk of infection related with bevacizumab for lung cancer patients. During the past 5 years, more prospective clinical trials with larger sample sizes have been performed, especially in NSCLC patients. We therefore pooled recent studies which could provide better and updated information on the overall risk and incidence of infection in NSCLC patients. The aim of this study is to gain a better understanding of the relative risk (RR) and overall incidence of infection in patients with NSCLC who received bevacizumab.

\section{Materials and Methods}

\section{Search Strategy}

A literature search was conducted using the following electronic databases, including PubMed, Embase, and Cochrane databases. Abstracts presented at the annual meetings of the American Society of Clinical Oncology (ASCO) were also searched. The upper date limit of March 31, 2021, was applied, with no lower date limit. Searches included the terms: ("bevacizumab" OR "Avastin") AND ("non-small cell lung cancer" OR "carcinoma of the lung") AND ("clinical trial" OR "randomized controlled trial"). The references cited by the included studies were also used to complete the search. Finally, the most updated package insert of bevacizumab was reviewed to identify relevant information.

\section{Study Selection}

Eligible criteria for inclusion in this meta-analysis were: (1) prospective phase II and III RCTs in NSCLC patients; (2) random assignment of participants to bevacizumab or the control (placebo or best supportive care) in addition to the same chemotherapy regimen and/or tyrosine kinase inhibitor; (3) English language; (4) data available regarding events or incidence of infection, including febrile neutropenia, unspecified infections, pneumonia, colitis, viral hepatitis, abscess, sepsis, and septic shock; and (5) in cases of duplicate publications, only the most complete, recent, and updated reports of the clinical trial were also included. Since bevacizumab was approved for the treatment of patients with cancer at a recommended dose of $5 \mathrm{mg} / \mathrm{kg}$ every 2 weeks or $7.5 \mathrm{mg} / \mathrm{kg}$ every 3 weeks, trials using bevacizumab at the approved dosage were included. Review articles, nonrandomized trials, and observational studies were excluded, i.e., phase I and single-arm phase II trials were omitted due to lack of controls. Primary studies with sample size less than 50 were excluded. Abstracts of all candidate articles were read by two independent readers (L.P. and B.D.Q.). Articles that could not be categorized based on the title and abstract alone were retrieved for full-text review. Disagreements were resolved by consensus between the two readers. To determine the issue of multiple publications from the same data sets, we checked all author names, clinical trial information, and the time period of patient recruitment of the articles. Two investigators (L.P. and B.D.Q.) independently assessed the eligibility of the articles and abstracts identified by the search, and discrepancies were resolved by consensus. Infectious complications were extracted from the safety and toxicity profile in the primary study. These clinical endpoints were all recorded according to Common Terminology Criteria for Adverse Events of National Cancer Institute (http://ctep.cancer. gov/reporting/ctc_archive.html). We included all incidences of infection of grade 1 or above in our analysis.

\section{Quality Assessment of Primary Studies}

The Jadad scoring system was used to assess the methodological quality of the included studies to evaluate the quality of factors. This provides a summary numeric quality score with a range of $0-5$. Two authors (L.P. and B.D.Q.) checked and confirmed the final results, and any disagreements were resolved by consensus with a third-party author (S.X.).

\section{Data Analysis}

A team of reviewers extracted the data from each study using a standardized form developed by the authors, including the following items: year of publication, first author, study name, number of patients, and treatment and control arms. If data from any of the above categories were not reported in the study, items were treated as "NR (not reported)." The data of the number of patients with all-grade and high-grade (grade 3 and above) infection receiving bevacizumab were extracted from the toxicity profile. We calculated and compared the RR of infection with the controls. For each study, we also derived the proportion and $95 \%$ confidence interval (CI) of patients with infection. For one study that reported zero events in the treatment or control arm, we applied the classic half-integer correction to calculate the RR and variance [4]. Between-study heterogeneity was estimated using the $\chi^{2}$-based $Q$ statistic [5]. Heterogeneity was considered statistically significant when $p<0.1$ or $I^{2}>50 \%$. If heterogeneity existed, data were analyzed using a random-effects model. In the absence of heterogeneity, a fixed-effects model was used. Incidence of infection was calculated using the number of patients experiencing infectious events and the total number of patients being treated. The number of patients for whom toxicity was evaluated was used as the number treated for each study. If the number of patients for whom toxicity was evaluated was not reported, the number of patients enrolled was used instead. To calculate the pooled incidence, an inverse variance statistical method was used. A statistical test with a $p$ value less than 0.05 was considered significant. To assess the stability of results, sensitivity analysis was carried out by sequential omission of individual studies. The presence of publication bias was evaluated by using Begg's and Egger's tests $[6,7]$. All of the calculations were performed by Stata version 14.0 (Stata Corporation, College Station, TX, USA).

\section{Results}

\section{Study Selection and Characteristics}

Our search strategy yielded a total of 463 potentially relevant articles on bevacizumab from the literature. After initial exclusion of duplicate and nonrandomized studies, 14 original studies [8-21] were considered eligible for the meta-analysis, comprising 4,545 patients for final analysis (Fig. 1). The major baseline characteristics of the 14 eligible studies were represented in Table 1, encompassing 8 phase III and 6 phase II clinical trials. The 


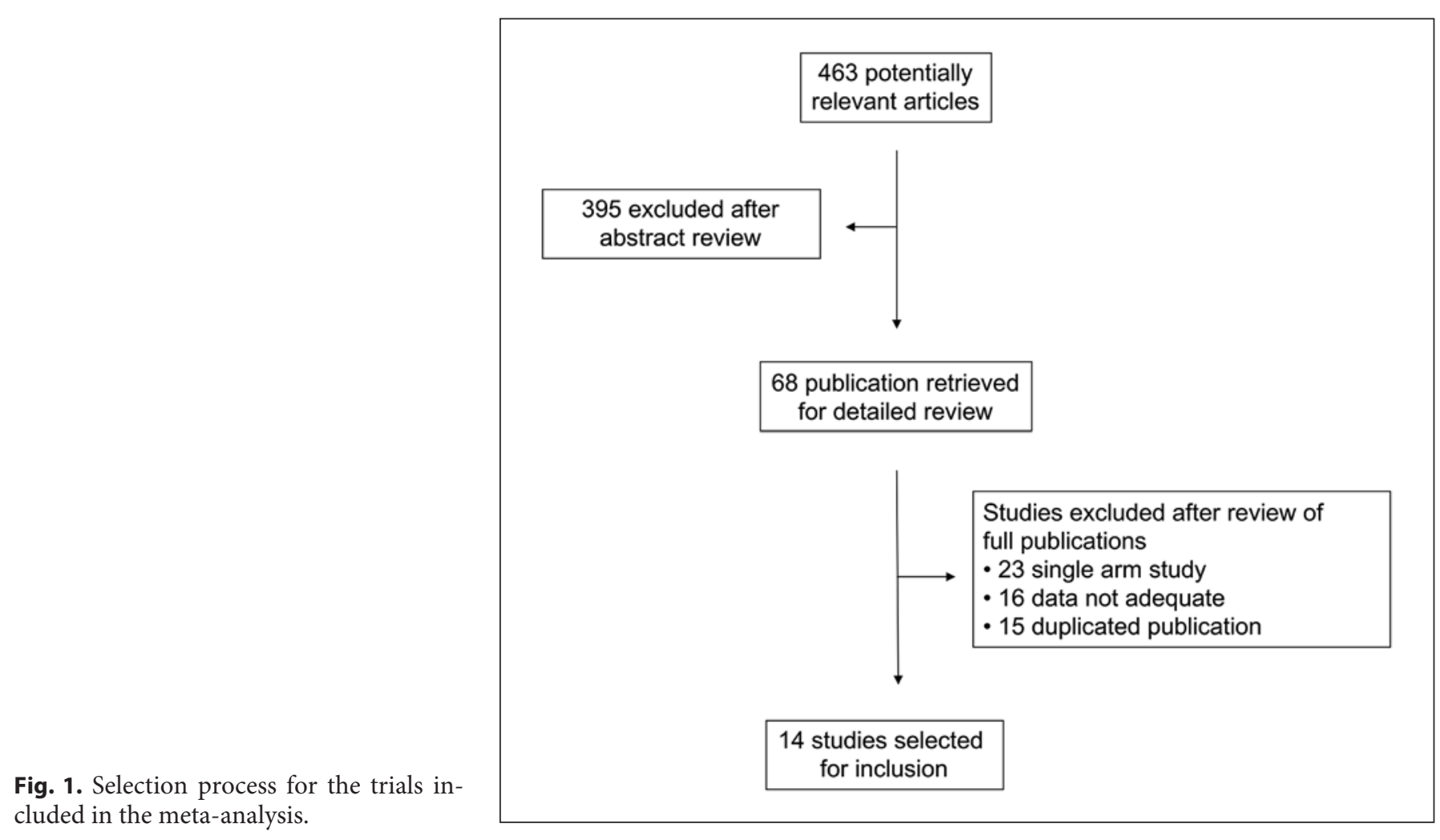

studies were published between 2004 and 2019. Studies were chosen and systemically reviewed in accordance with the PRISMA statement [22]. The Jadad scores ranged from 3 to 5 . No trial received a low-quality Jadad score (i.e., 1-2), confirming our selection criteria.

\section{$R R$ of Infection}

RR was calculated to determine the particular contribution of bevacizumab to infection by eliminating the influence of confounding factors, such as comorbidities and other concomitant medications. Data on infection were available from 14 trials, representing a total of 4,545 patients (Table 1). All 14 studies reported RR of highgrade infection, while 5 studies $[8,14,16,20,21]$ reported RR of all-grade infection. There was no significant heterogeneity $\left(p=0.989 ; I^{2}=0.0 \%\right)$ for the all-grade RR analysis. The pooled RR showed that bevacizumab treatment did not increase the risk of developing all-grade infections in NSCLC patients with a RR of 1.12 (95\% CI: 0.84-1.49, Fig. 2a). The RR for high-grade infection was also not increased in NSCLC patients receiving bevacizumab compared with the control $(\mathrm{RR}=1.11,95 \% \mathrm{CI}$ : $0.86-1.41$, Fig. $2 b)$ without significant heterogeneity $(p=$ $\left.0.477 ; I^{2}=0.0 \%\right)$. Exploratory subgroup analyses indicated that when grouped according to the phase, treatment line, ethnic group (Asian vs. non-Asian), and concomitant drug with bevacizumab, respectively, the RR for high-grade infection remained similar between subgroups (Table 2).

Infection with Bevacizumab in NSCLC

\section{Incidence of Infection}

The summary incidences of all-grade and high-grade infection in patients receiving bevacizumab in the treatment group were $16.4 \%$ (95\% CI: 15.7-17.2\%) and 4.3\% (95\% CI: 3.0-6.1\%), respectively (Fig. 3a, b). While high-grade incidence was grouped according to the subgroup of the phase of the clinical trial, treatment line, and ethnic population, the results were similar (Table 2). When bevacizumab was used in combination with different anticancer drugs, e.g., EGFR tyrosine kinase inhibitors (TKIs) versus chemotherapy, the incidence of high-grade infection was lower in the TKI group than in that of the chemotherapy group. We also calculated the all-grade and high-grade incidence of infection in the control group, resulting in 16.4\% (95\% CI: $10.8-$ $24.8 \%$ ) and 3.6\% (95\% CI: 2.0-6.4\%), respectively (Fig. 4).

\section{Publication Bias}

Begg's funnel plot and Egger's test were performed to evaluate the publication bias of the eligible studies. All 14 studies investigating high-grade risk infection associated with bevacizumab yielded an Egger's test score of $p=0.838$, indicating the absence of publication bias in the studies (Fig. 5a).

\section{Sensitivity Analysis}

Sensitivity analysis was performed to examine the stability and reliability of the pooled RRs by sequential omission of individual studies. Our results indicated that risk of high-grade infection with bevacizumab was shown to be statistically stable (Fig. 5b). 


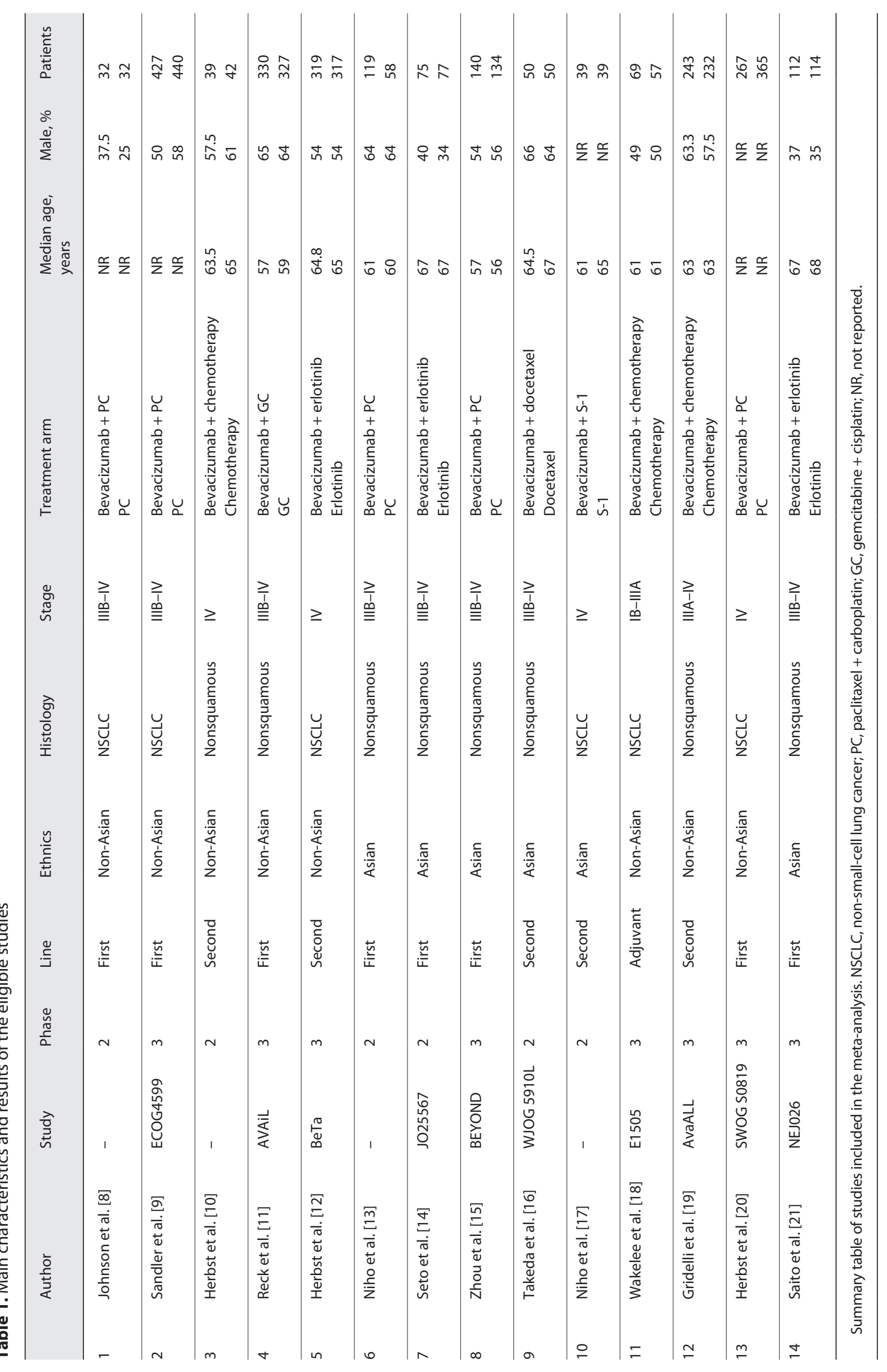




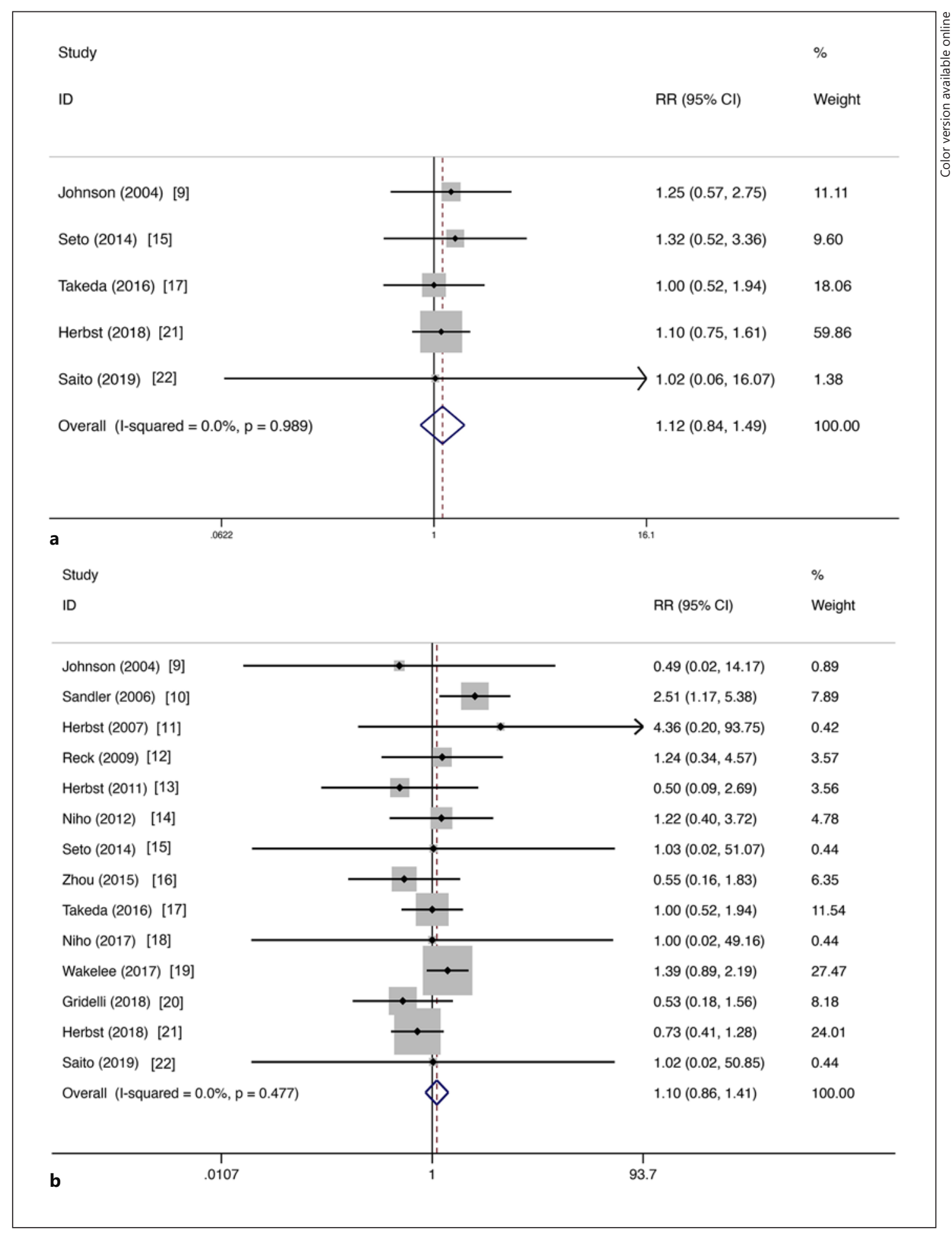

Fig. 2. Forest plot of RR of all-grade and high-grade infections associated with bevacizumab versus the control. Each study was shown by the name of the lead author and year of publication. For study name, the first author's name was used to represent each trial. RR of bevacizumab-associated all-grade and high-grade infections versus the control from controlled trials of cancer patients. Overall summary RR of infection was calculated using a fixed-ef- fects or random-effects model. RR for each study is displayed numerically on the left and graphically on the right. For each trial the position of the square denoted the value of $\mathrm{RR}$, horizontal lines represent $95 \% \mathrm{CI}$, and diamond plot represents overall results of the included trials. Plots are arranged as follows: RR of all-grade infection (a) and RR of high-grade infection (b). 
Table 2. Subgroup analysis of risk and incidence of high-grade infection with bevacizumab

\begin{tabular}{|c|c|c|c|c|c|c|c|}
\hline & $\begin{array}{l}\text { Studies, } \\
n\end{array}$ & $\begin{array}{l}\text { RR } \\
(95 \% \mathrm{Cl})\end{array}$ & $R^{2}, \%$ & $\begin{array}{l}p \text { value for } \\
\text { heterogeneity }\end{array}$ & $\begin{array}{l}\text { Incidence } \\
(95 \% \mathrm{Cl})\end{array}$ & $R^{2}, \%$ & $\begin{array}{l}p \text { value } \\
\text { for heterogeneity }\end{array}$ \\
\hline \multicolumn{8}{|l|}{ Line } \\
\hline First & 8 & $1.08(0.75-1.55)$ & 13.3 & 0.33 & $0.04(0.03-0.06)$ & 91.0 & 0.00 \\
\hline Second & 5 & $0.83(0.49-1.38)$ & 0.0 & 0.65 & $0.03(0.01-0.18)$ & 97.9 & 0.00 \\
\hline Adjuvant & 1 & $1.39(0.89-2.19)$ & - & - & $0.06(0.06-0.06)$ & - & - \\
\hline \multicolumn{8}{|l|}{ Phase } \\
\hline 3 & 8 & $1.10(0.84-1.46)$ & 39.2 & 0.12 & $0.04(0.03-0.04)$ & 93.5 & 0.00 \\
\hline 2 & 6 & $1.11(0.65-1.90)$ & 0.0 & 0.95 & $0.08(0.03-0.20)$ & 94.5 & 0.00 \\
\hline \multicolumn{8}{|l|}{ Ethnic } \\
\hline Asian & 6 & $0.92(0.56-1.53)$ & 0.0 & 0.96 & $0.06(0.02-0.16)$ & 96.3 & 0.00 \\
\hline Non-Asian & 8 & $1.16(0.88-1.54)$ & 37.1 & 0.13 & $0.04(0.03-0.05)$ & 92.9 & 0.00 \\
\hline \multicolumn{8}{|l|}{ Drug } \\
\hline Chemotherapy & 11 & $1.13(0.88-1.45)$ & 15.1 & 0.30 & $0.05(0.04-0.07)$ & 0.00 & 0.98 \\
\hline TKI & 3 & $0.60(0.14-2.50)$ & 0.0 & 0.91 & $0.01(0.00-0.02)$ & 97.9 & 0.00 \\
\hline
\end{tabular}

\section{Discussion}

The present meta-analysis has combined 14 publications including 8 phase III and 6 phase II trials. Our meta-analysis results demonstrate that bevacizumab was not associated with an increased risk of developing infection. The RR of infection of bevacizumab compared to controls was numerically increased but without statistical significance. The overall incidences of all-grade and high-grade infection were $16.4 \%$ and $4.3 \%$, respectively. In subgroup analyses for high-grade infection, we analyzed the concomitant drugs such as TKIs and chemotherapy, two combination options routinely used in clinical practice for NSCLC. As expected, combination with chemotherapy resulted in a higher incidence than with TKIs; however, in terms of RR, subgroup analysis of highgrade risk between concomitant drugs remained similar.

In a previous systematic review by Rafailidis et al. [2], due to the limited number of RCTs, they were unable to quantitatively analyze the risk and incidence of infection of bevacizumab. In 2015, a meta-analysis investigating the risk and incidence of bevacizumab in cancer patients demonstrated that the use of bevacizumab is related to a higher risk of infectious complications in patients with all cancers [3]. This meta-analysis only incorporated 4 trials of NSCLC [8, $9,11,12]$; the risk of high-grade infection varied significantly with concomitant drugs and underlying malignancy. Hepatocellular carcinoma has the lowest risk of infection $(\mathrm{RR}=0.96,95 \% \mathrm{CI}, 0.16-5.77)$, while gastric cancer was associated with the highest risk ( $\mathrm{RR}=2.43,95 \% \mathrm{CI}, 1.29$ 4.58). Another previous meta-analysis conducted by Zhou et al. [23] found a significantly increased risk of high-grade neutropenia (RR 1.10, 95\% CI: 1.02-1.19) and high-grade febrile neutropenia (RR 1.31, 95\% CI: 1.08-1.59) in patients treated with bevacizumab. Among the 18 trials analyzed, 3 studies of NSCLC were included [9-11]. The results also indicated that bevacizumab-related febrile neutropenia varied significantly among tumor types, which raised the question regarding whether underlying tumor biology might affect the incidence of adverse events.

Our meta-analysis has included NSCLC patients ranging from stage I to IV, and the studied population is representative for the whole population of NSCLC patients. Although bevacizumab is approved to treat advanced nonsquamous NSCLC, previous clinical trial investigated the role of bevacizumab in a neoadjuvant setting, which did not improve downstaging in unselected patients [24]. Likewise, the addition of bevacizumab in adjuvant setting did not confer overall survival benefit in NSCLC patients [18]. The infectious toxicity of bevacizumab in neoadjuvant and adjuvant settings is of special importance as drugs shall not impact surgical complication rates. Although the included studies are not sufficient to draw a conclusion for perioperative setting, we did not observe an elevated risk of infection in the subgroup analysis in our meta-analysis. Some of the clinical trials have incorporated lung squamous-cell carcinoma patients, but we could not discriminate whether squamous lung cancer patients will have a higher risk of infection related with bevacizumab.

We attempted to find whether different concomitant drugs are risk factors for infections associated with bevacizumab and found there were no significant differences in the risk of infection. When combined with chemotherapy, bevacizumab has a numerically higher incidence than that with TKIs, which may due to the higher incidence of infection due to chemotherapeutic drugs. The future application of bevacizumab in NSCLC has moved to combination with immunotherapy drugs, such as immune checkpoint inhibitors. The risk of bevacizumabrelated infection shall be taken into consideration when combination therapy is used in clinical practice.

The mechanism of bevacizumab-related infection is not entirely clear. Neutropenia could be a possible mech- 


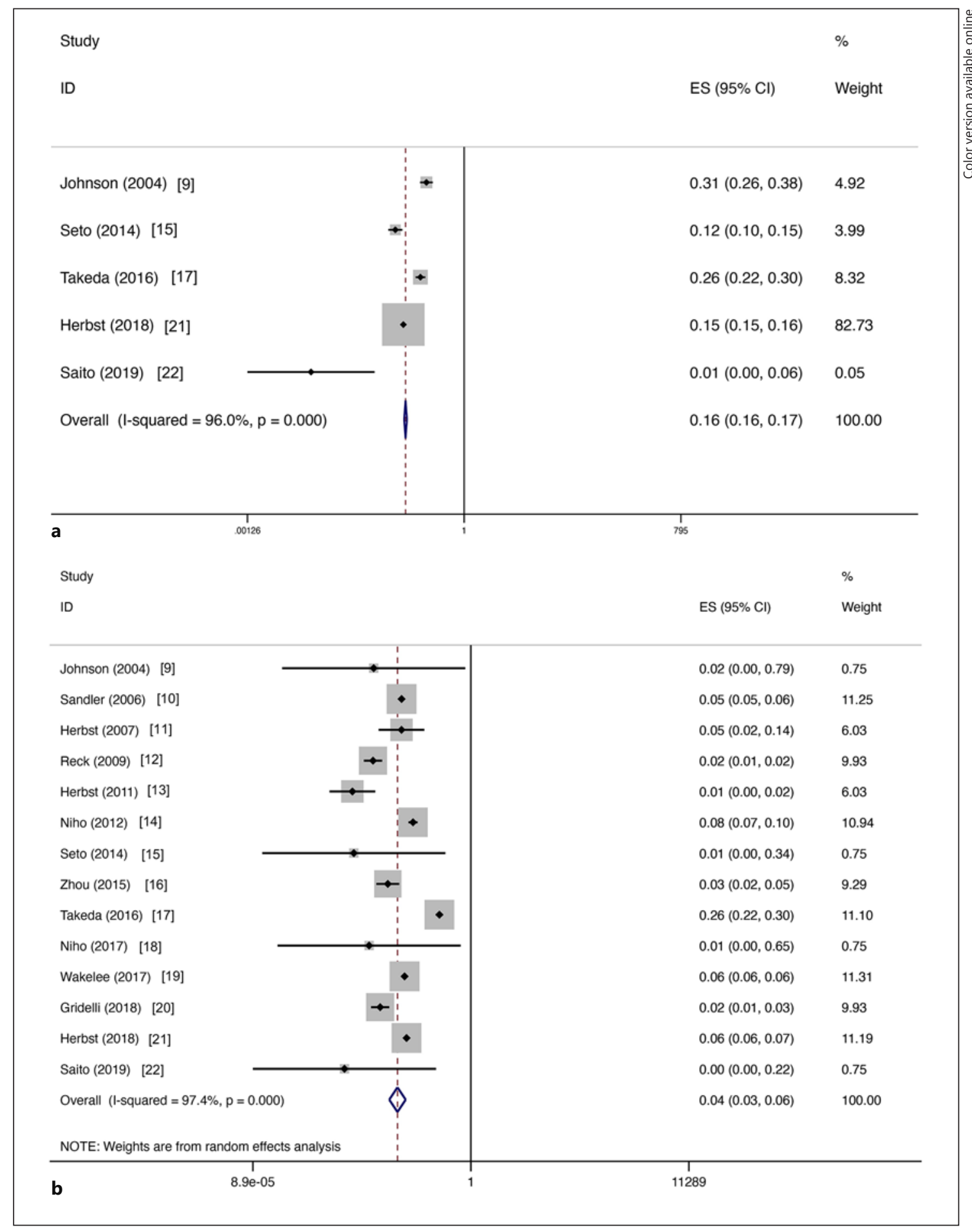

Fig. 3. Forest plot of the incidence of all-grade and high-grade infections in the bevacizumab group. Each study was shown by the name of the lead author and year of publication. For study name, the first author's name was used to represent each trial. The inci- dence of bevacizumab group for all-grade and high-grade infections. Plots are arranged as follows: the incidence of all-grade infection (a) and incidence of high-grade infection (b). anism that contributes to the risk of infection related with bevacizumab [25], which is also one of the reasons of treatment-related mortality with bevacizumab in cancer patients [26]. Further data are still needed to characterize the real risk and incidence of infection in patients with
NSCLC receiving bevacizumab as part of their treatment regimens. This applies across the board as other antiangiogenesis drugs were reported to increase the risk of infection, including aflibercept [27] and VEGFR TKI inhibitors [28]. 


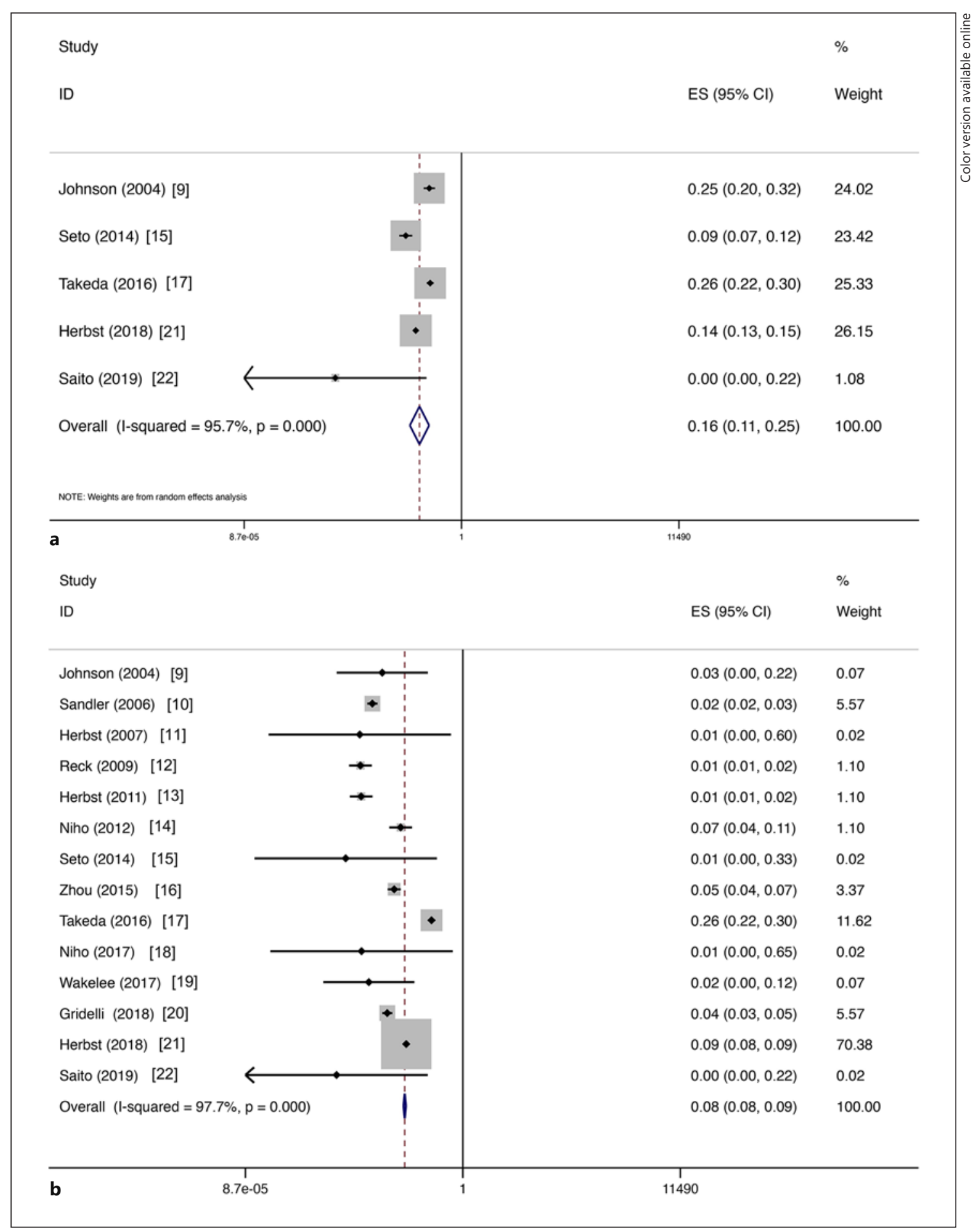

Fig. 4. Forest plot of the incidence of all-grade and high-grade infections in the control group. Each study was shown by the name of the lead author and year of publication. For study name, the first author's name was used to represent each trial. The incidence of the control group for all-grade and high-grade infections. Plots are arranged as follows: the incidence of all-grade infection (a) and incidence of high-grade infection (b).
Our meta-analysis has several limitations. First, although we analyzed the risk and incidence of high-grade infection in the subgroup of concomitant drugs with bevacizumab, multiple chemotherapy regimens given in the trials may limit the interpretation of our results. Second, the trials included in our study might have underestimated the incidence and risk of bevacizumab-associated infection because of the use of different versions of adverse event reporting criteria. Toxicity data in RCTs have been reported to be suboptimal and variable as toxicity is usu- 
Fig. 5. Publication bias (a) and sensitivity analysis (b) of RR of bevacizumab-associated all-grade infection versus the control.

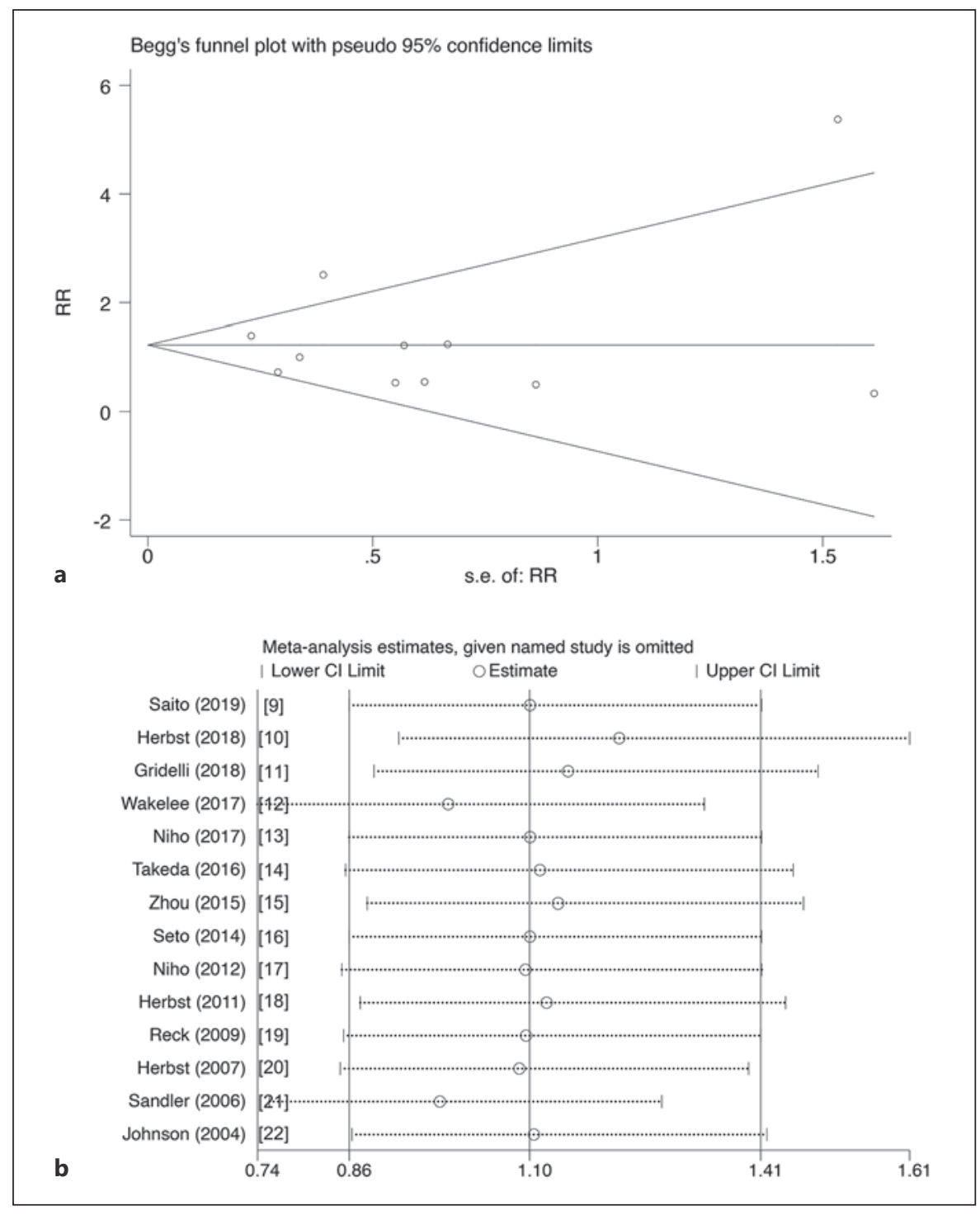

ally not the primary outcome measure [29]. Specific etiologies of infection were not reported in many of the included RCTs. Therefore, low incidence AEs would be somewhat overlooked in clinical trials. Third, there is significant heterogeneity of incidence of all-grade infection with bevacizumab-based regimen, although subgroup analysis of concomitant drugs can partially explain heterogeneity along with the different trial designs.

\section{Conclusions}

Our meta-analysis is the first study to systematically estimate the RR and incidence of infection associated with anti-VEGF monoclonal antibody bevacizumab in NSCLC patients. Contrary to previous meta-analyses results across cancer, our data showed that bevacizumab was not associated with a higher risk of developing infection compared with controls in NSCLC.

Infection with Bevacizumab in NSCLC

\section{Acknowledgments}

We are indebted to the authors of the primary studies.

\section{Statement of Ethics}

An ethics statement was not required for this study type as no human or animal subjects or materials were used.

\section{Conflict of Interest Statement}

J.S. refers to his conflicts of interest which are not relevant here at https://www.nature.com/onc/editors. Other authors: none declared.

\section{Funding Sources}

This study was partially supported by the Natural Science Foundation of Zhejiang Province, China (Grant No.: LY19H160041). The funders had no role in study design, data collection and analysis, decision to publish, or preparation of the manuscript. 


\section{Author Contributions}

Conceptualization: L.P. and J.S.; data analysis: L.P., S.X., Y.X., and J.S.Y.; writing - original draft preparation: L.P., B.D.Q., and K.X.; writing - review and editing: L.P., Y.X., and J.S.; supervision: J.S.; funding acquisition: L.P. All the authors have read and agreed to the published version of the manuscript.

\section{Data Availability Statement}

All data generated or analyzed during this study are included in this article. Further inquiries can be directed to the corresponding author.

\section{References}

1 Kabbinavar FF, Ellis LM. Can inhibition of angiogenic pathways increase the efficacy of intravenous 5-fluorouracil-based regimens? Clin Colorectal Cancer. 2004 Oct; 4 Suppl 2: S69-73.

2 Rafailidis PI, Kakisi OK, Vardakas K, Falagas ME. Infectious complications of monoclonal antibodies used in cancer therapy: a systematic review of the evidence from randomized controlled trials. Cancer. 2007 Jun 1;109(11): 2182-9.

3 Qi WX, Fu S, Zhang Q, Guo XM. Bevacizum$\mathrm{ab}$ increases the risk of infections in cancer patients: a systematic review and pooled analysis of 41 randomized controlled trials. Crit Rev Oncol Hematol. 2015 Jun;94(3):323-36.

4 Choueiri TK, Schutz FA, Je Y, Rosenberg JE, Bellmunt J. Risk of arterial thromboembolic events with sunitinib and sorafenib: a systematic review and meta-analysis of clinical trials. J Clin Oncol. 2010 May 1;28(13):2280-5.

5 Zintzaras E, Ioannidis JP. Heterogeneity testing in meta-analysis of genome searches. Genet Epidemiol. 2005 Feb;28(2):123-37.

6 Yusuf S, Peto R, Lewis J, Collins R, Sleight P. Beta blockade during and after myocardial infarction: an overview of the randomized trials. Prog Cardiovasc Dis. 1985 Mar-Apr; 27(5):335-71

7 Begg CB, Mazumdar M. Operating characteristics of a rank correlation test for publication bias. Biometrics. 1994 Dec;50(4):1088-101.

8 Johnson DH, Fehrenbacher L, Novotny WF, Herbst RS, Nemunaitis JJ, Jablons DM, et al. Randomized phase II trial comparing bevacizumab plus carboplatin and paclitaxel with carboplatin and paclitaxel alone in previously untreated locally advanced or metastatic nonsmall-cell lung cancer. J Clin Oncol. 2004 Jun 1;22(11):2184-91.

9 Sandler A, Gray R, Perry MC, Brahmer J, Schiller JH, Dowlati A, et al. Paclitaxel-carboplatin alone or with bevacizumab for nonsmall-cell lung cancer. N Engl J Med. 2006 Dec 14;355(24):2542-50.

10 Herbst RS, O’Neill VJ, Fehrenbacher L, Belani CP, Bonomi PD, Hart L, et al. Phase II study of efficacy and safety of bevacizumab in combination with chemotherapy or erlotinib compared with chemotherapy alone for treatment of recurrent or refractory non small-cell lung cancer. J Clin Oncol. 2007 Oct 20;25(30): 4743-50.

11 Reck M, von Pawel J, Zatloukal P, Ramlau R, Gorbounova V, Hirsh V, et al. Phase III trial of cisplatin plus gemcitabine with either placebo or bevacizumab as first-line therapy for nonsquamous non-small-cell lung cancer:
AVAil. J Clin Oncol. 2009 Mar 10;27(8): 1227-34.

12 Herbst RS, Ansari R, Bustin F, Flynn P, Hart L, Otterson GA, et al. Efficacy of bevacizumab plus erlotinib versus erlotinib alone in advanced non-small-cell lung cancer after failure of standard first-line chemotherapy (BeTa): a double-blind, placebo-controlled, phase 3 trial. Lancet. 2011 May 28;377(9780): 1846-54.

13 Niho S, Kunitoh H, Nokihara H, Horai T, Ichinose Y, Hida T, et al. Randomized phase II study of first-line carboplatin-paclitaxel with or without bevacizumab in Japanese patients with advanced non-squamous non-small-cell lung cancer. Lung Cancer. 2012 Jun;76(3): 362-7.

14 Seto T, Kato T, Nishio M, Goto K, Atagi S, Hosomi Y, et al. Erlotinib alone or with bevacizumab as first-line therapy in patients with advanced non-squamous non-small-cell lung cancer harbouring EGFR mutations (JO25567): an open-label, randomised, multicentre, phase 2 study. Lancet Oncol. 2014 Oct;15(11):1236-44.

15 Zhou C, Wu YL, Chen G, Liu X, Zhu Y, Lu S, et al. BEYOND: a randomized, double-blind, placebo-controlled, multicenter, phase III study of first-line carboplatin/paclitaxel plus bevacizumab or placebo in chinese patients with advanced or recurrent nonsquamous non-small-cell lung cancer. J Clin Oncol. 2015 Jul 1;33(19):2197-204.

16 Takeda M, Yamanaka T, Seto T, Hayashi H, Azuma K, Okada M, et al. Bevacizumab beyond disease progression after first-line treatment with bevacizumab plus chemotherapy in advanced nonsquamous non-small cell lung cancer (West Japan Oncology Group 5910L): an open-label, randomized, phase 2 trial. Cancer. 2016 Apr 1;122(7):1050-9.

17 Niho S, Ohe Y, Ohmatsu H, Umemura S, Matsumoto S, Yoh K, et al. Switch maintenance chemotherapy using S-1 with or without bevacizumab in patients with advanced non-small cell lung cancer: a phase II study. Lung Cancer. 2017 Jun;108:66-71.

18 Wakelee HA, Dahlberg SE, Keller SM, Tester WJ, Gandara DR, Graziano SL, et al. Adjuvant chemotherapy with or without bevacizumab in patients with resected non-small-cell lung cancer (E1505): an open-label, multicentre, randomised, phase 3 trial. Lancet Oncol. 2017 Dec;18(12):1610-23.

19 Gridelli C, de Castro Carpeno J, Dingemans AC, Griesinger F, Grossi F, Langer C, et al. Safety and efficacy of bevacizumab plus standard-of-care treatment beyond disease pro- gression in patients with advanced non-small cell lung cancer: the AvaALL randomized clinical trial. JAMA Oncol. 2018 Dec 1;4(12): e183486.

20 Herbst RS, Redman MW, Kim ES, Semrad TJ, Bazhenova L, Masters G, et al. Cetuximab plus carboplatin and paclitaxel with or without bevacizumab versus carboplatin and paclitaxel with or without bevacizumab in advanced NSCLC (SWOG S0819): a randomised, phase 3 study. Lancet Oncol. 2018 Jan;19(1):101-14.

21 Saito H, Fukuhara T, Furuya N, Watanabe K, Sugawara S, Iwasawa S, et al. Erlotinib plus bevacizumab versus erlotinib alone in patients with EGFR-positive advanced nonsquamous non-small-cell lung cancer (NEJ026): interim analysis of an open-label, randomised, multicentre, phase 3 trial. Lancet Oncol. 2019 May;20(5):625-35.

22 Moher D, Liberati A, Tetzlaff J, Altman DG Preferred reporting items for systematic reviews and meta-analyses: the PRISMA statement. BMJ. 2009 Jul 21;339(7):b2535.

23 Zhou F, Shao JH, Wu LQ, Yin XB, Yu X. Risk of serious neutropenic events in cancer patients treated with bevacizumab: a meta-analysis. Asian Pac J Cancer Prev. 2014;14(4):2453-9.

24 Chaft JE, Rusch V, Ginsberg MS, Paik PK, Finley DJ, Kris MG, et al. Phase II trial of neoadjuvant bevacizumab plus chemotherapy and adjuvant bevacizumab in patients with resectable nonsquamous non-small-cell lung cancers. J Thorac Oncol. 2013 Aug;8(8): 1084-90.

25 Schutz FA, Jardim DL, Je Y, Choueiri TK. Haematologic toxicities associated with the addition of bevacizumab in cancer patients. Eur J Cancer. 2011 May;47(8):1161-74.

26 Ranpura V, Hapani S, Wu S. Treatment-related mortality with bevacizumab in cancer patients: a meta-analysis. JAMA. 2011 Feb 2; 305(5):487-94.

27 Zhang X, Ran Y, Shao Y, Wang K, Zhu Y. Incidence and risk of severe infections associated with aflibercept in cancer patients: a systematic review and meta-analysis. Br J Clin Pharmacol. 2016 Jan;81(1):33-40.

$28 \mathrm{Ma}$ Q, Gu LY, Ren YY, Zeng LL, Gong T, Zhong DS. Increased risk of severe infections in cancer patients treated with vascular endothelial growth factor receptor tyrosine kinase inhibitors: a meta-analysis. Onco Targets Ther. 2015;8:2361-74.

29 Seruga B, Sterling L, Wang L, Tannock IF. Reporting of serious adverse drug reactions of targeted anticancer agents in pivotal phase III clinical trials. J Clin Oncol. 2011 Jan 10;29(2): 174-85. 